\title{
Fabrication of Lotus Nickel Through Thermal Decomposition Method of Compounds under Ar Gas Atmosphere
}

\author{
Sang-Youl Kim ${ }^{\dagger}$, Bo-Young Hur and Hideo Nakajima* \\ ERI, i-Cube Center, Gyeongsang National University, 900 Kajoa-Dong Jinju 660-701 Korea \\ *The Institute of Scientific and Industrial Research, Osaka University, 8-1 Mihogaoka, Ibaraki, Osaka, 567-0047, Japan
}

(Received January 9, 2009 : Received in revised form April 29, 2009 : Accepted April 29, 2009)

\begin{abstract}
Lotus-type porous nickel with cylindrical pores was fabricated by unidirectional solidification under an $\mathrm{Ar}$ gas atmosphere using the thermal decomposition method of the compounds such as sodium hydroxide, calcium hydroxide, calcium carbonate, and titanium hydride. The decomposed gas does form the pores in liquid nickel, and then, the pores become the cylindrical pores during unidirectional solidification. The decomposed particles from the compounds do play a rule on nucleation sites of the pores. The behavior of pore growth was controlled by atmosphere pressure, which can be explained by Boyle's law. The porosity and pore size decreased with increasing Ar gas pressure when the pores contain hydrogen gas decomposed from calcium and sodium hydroxide and titanium hydride, ; however it they did not change when the pores contain containing carbon dioxide decomposed from calcium carbonate. These results indicate that nickel does not have the solubility of carbon dioxide. Lotus-type porous metals can be easily fabricated by the thermal decomposition method, which is superior to the conventional fabrication method used to pressurized gas atmospheres.
\end{abstract}

Key words lotus-type porous metal, thermal decomposition method, compound, hydrogen pores.

\section{Introduction}

Porous metal is one of the emerging materials in the field of industrial applications such as automotive, catalyst and transport. However, due to its various special properties such as superior light-weight, good energy absorption, high stiffness and outstanding damping capability, it has been known as one of the most applicable material for many structural and functional applicable in such various fields of automotives, transports, ships and aerospace. Especially, nickel foams are well-suited for battery applications and are involved for instance in portable computers and mobile phones. This requires outstanding mechanical and electrical properties. The use of lightweight and highly conductive foams lead to a considerable increase of the energy density, whereas a high tensile strength is necessary for smooth processing of the foam during the battery production steps like pasting, calendering and coiling. ${ }^{1)}$

Conventional porous metals with spherical pores are fabricated by using foaming agents such as titanium hydride and calcium carbonate containing gas molecular. ${ }^{2,3,4)}$

Corresponding author

E-Mail : ulsfom@naver.com (S. Y. Kim)
These porous metals have a closed pore and high porosity, although they are detrimental to mechanical strength. For industrial application, a homogeneous pore structure of porous metals without damaging to properties is necessary. Recently, several investigators ${ }^{5,6,7)}$ have studied the fabrication of porous metals whose long cylindrical pores are aligned in one direction by a unidirectional solidification method under pressurized hydrogen gas. Lotus-type porous metal [lotus metal] has excellent properties such as sound absorption, damping capacity, high thermal conductivity and bio-compatibility. Also, lotus metals have superior mechanical properties to metal foam, which is fabricated by casting method or powder metallurgy. $8,9,10)$

Lotus metals are fabricated by utilizing gas solubility gap between solid and liquid state under high pressurized gas atmosphere. ${ }^{5)}$ Ide et al. ${ }^{11)}$ reported the fabrication of lotus-type porous nickel-based intermetallics under pressurized hydrogen gas atmosphere. The porosity and pore size of lotus metals depends on atmospheric pressure such as hydrogen, nitrogen and oxygen. However, hydrogen gas is inflammable and explosive when it is mixed with oxygen. It is necessary to more stable and simple fabrication method for fabricating lotus metals. Also Onishi et al. ${ }^{12)}$ reported the fabrication of lotus type porous 
nickel using moisture in the argon gas atmosphere. In this case, it was difficult to control the moisture content in the atmosphere and the mold with coated ceramic layer. Therefore, it is desirable to develop an easy and safe process for fabricating lotus metals. Nakajima and Ide $^{13)}$ proposed to thermal decomposition method [TDM] using the compounds containing gas element. They reported to fabricate lotus copper with elongated pores using titanium hydride under Ar gas atmosphere. Because lotus-type porous copper using titanium hydride has homogeneous pore structure, it is tried to fabricate lotustype porous nickel by thermal decomposition method. In this paper, we have fabricated lotus nickel using four kinds of compounds and compared the porosity and pore size under Ar gas atmosphere.

\section{Experimental Procedures}

Lotus nickel was fabricated from $99.9 \%$ pure electrolytic nickel. For removing the surface oxide of electrolytic nickel was carried out by grinding, followed

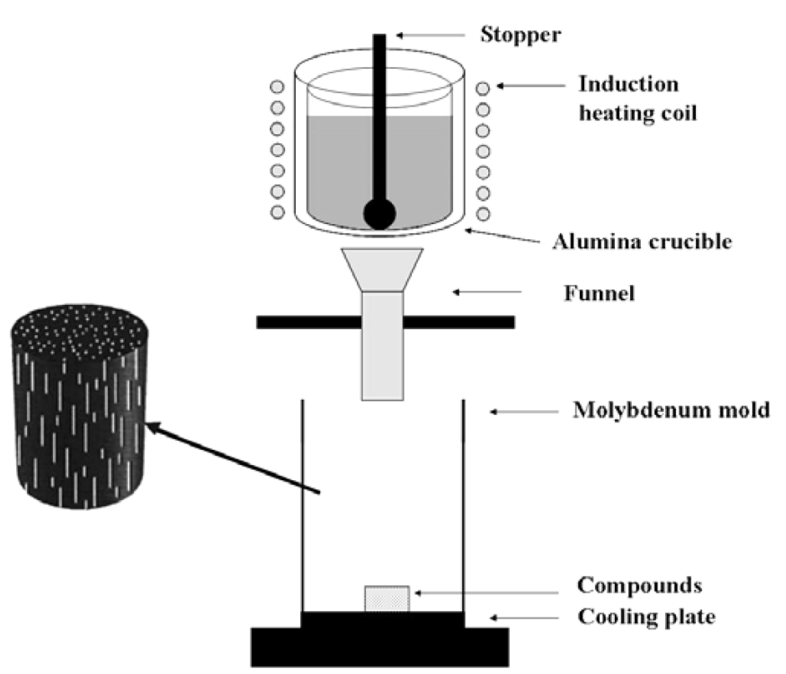

Fig. 1. Mold casting technique for fabrication of lotustype porous nickel. by ultrasonic cleaning in acetone. About $150 \mathrm{~g}$ of nickel is weighed out and used as the constituent for lotus nickel. A schematic drawing of the fabrication apparatus of lotus type porous metals using compounds is shown in Fig. 1. This apparatus consists of one part is melting the materials and another part is solidifying the molten materials. The alumina crucible and the mold were set up in the chamber. The chamber was evacuated to several $\mathrm{Pa}$ by a rotary pump. Then, Ar gas is filled in the chamber from $0.1 \mathrm{MPa}$ to $1.0 \mathrm{MPa}$. A copper plate was assembled on the bottom of the water cooled mold. A molybdenum sheet with a thickness of $0.1 \mathrm{~mm}$ was formed on a cylinder with an inner diameter of approximately $30 \mathrm{~mm}$ and a height of approximately $90 \mathrm{~mm}$ as the side wall of the mold. Boron nitride was coated to the inside of the molybdenum mold. The compounds containing gas elements used to the pore evolution source with different gas source. The compound was put on a copper plate. Nickel was heated in alumina crucible set up inside the high-frequency induction coil in the melting pot. The temperature of the liquid in the alumina crucible was monitored by an infrared pyrometer (model IR-AP, Chino Co.). Molten nickel in the alumina crucible was poured into the mold and solidified in one direction.

An ingot of lotus-type porous nickel was cut parallel and perpendicular to the solidified direction by a sparkerosion wire cutting machine (Model A320D, Sodic Co.) for observing the pore morphology. For the characterization of pore structure, samples mechanically ground with 2002400 grit grinding paper. The shape and size of pores was observed with an optical microscope and evaluated with image analysis (Win ROOF, Mitani Co.). The porosity (p) was evaluated from the following equation:

$$
\begin{aligned}
\mathrm{p}(\%)= & \left(1-\frac{\text { Apparent density of porous aluminum }}{\text { Desity of nonporous aluminum }}\right) \\
& \times 100
\end{aligned}
$$

Table 1. Decomposition reactions and temperature of each compounds.

\begin{tabular}{cccc}
\hline Reactions & Decomposition temperature $[\mathrm{K}]$ & Gas atoms to be dissolved & Ref. \\
\hline $\begin{array}{c}2 \mathrm{NaOH} \rightarrow \mathrm{Na}_{2} \mathrm{O}+\mathrm{H}_{2} \mathrm{O} \\
\mathrm{H}_{2} \mathrm{O} \rightarrow \text { metallic oxide }+\mathrm{H}_{2}\end{array}$ & 1663 & $\mathrm{H}_{2}$ & {$[14]$} \\
$\mathrm{Ca}(\mathrm{OH})_{2} \rightarrow \mathrm{CaO}+\mathrm{H}_{2} \mathrm{O}$ & & & \\
$\mathrm{H}_{2} \mathrm{O} \rightarrow$ metallic oxide $+\mathrm{H}_{2}$ & 853 & $\mathrm{H}_{2}$ & {$[15]$} \\
$\mathrm{TiH}_{2} \rightarrow \mathrm{Ti}+\mathrm{H}_{2}$ & 723 & $\mathrm{H}_{2}$ & {$[16]$} \\
$\mathrm{CaCO}_{3} \rightarrow \mathrm{CaO}+\mathrm{CO}_{2}$ & 973 & $\mathrm{CO}_{2}$ & {$[17]$} \\
\hline
\end{tabular}




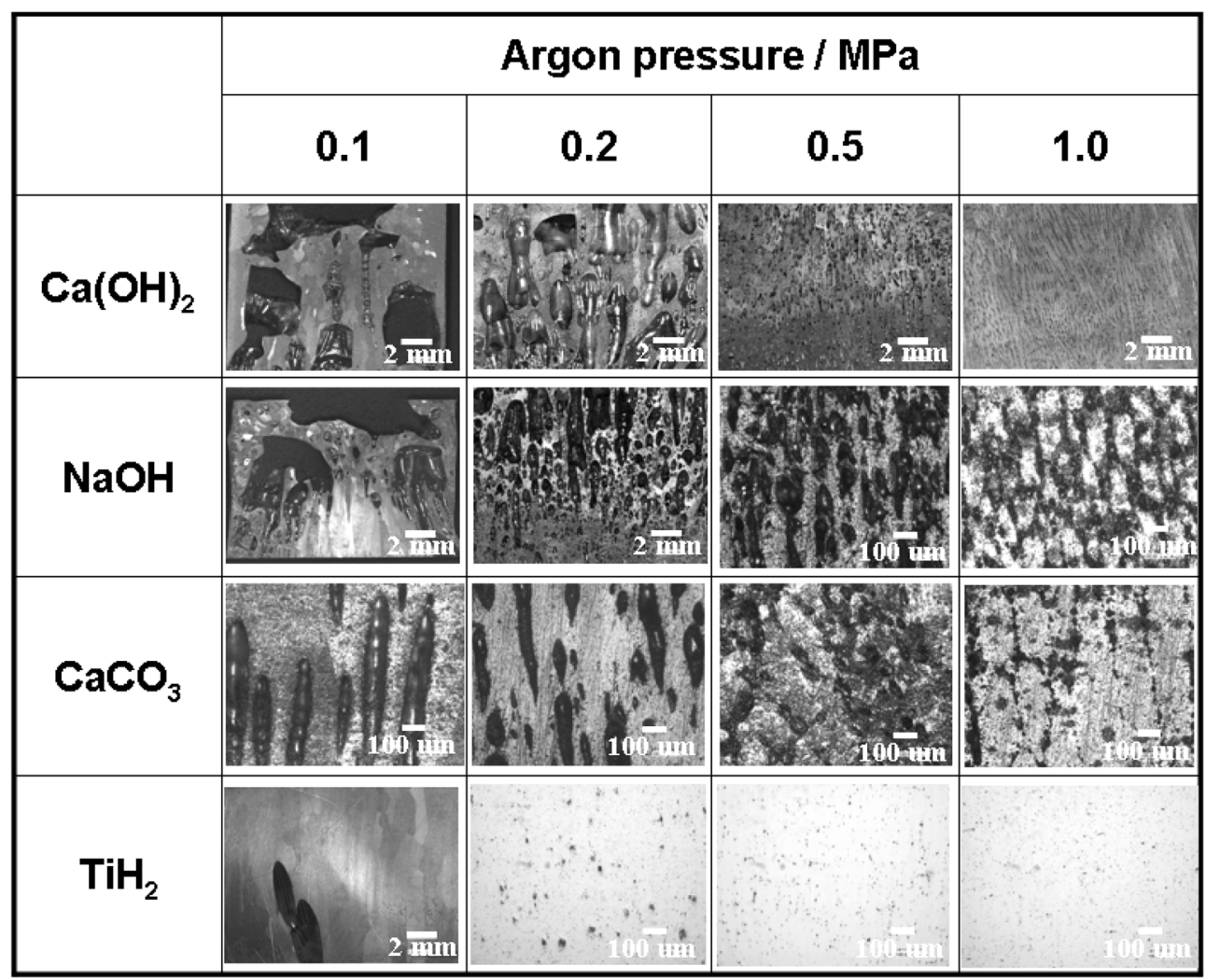

Fig. 2. Pore structure of lotus nickel fabricated through thermal decomposition parallel to solidification direction.

The apparent density of the individual specimen was calculated by measuring both the weight and the apparent volume of each specimen.

\section{Results and Discussion}

Four kinds of gas releasing compounds such as sodium hydroxide, titanium hydride and calcium carbonate were used. Table 1 shows the decomposition reactions and temperature of different compounds which released gas such as vapour, hydrogen and carbon dioxide. Each compound was decomposed when the melt was poured into the mold. The compounds were decomposed to gas and oxide or metals. Decomposed gases formed the pores in the solid and the oxides became pore nucleation sites. The pores are aligned by unidirectional solidification in metal when the melt is quickly solidified by cooled chiller.

Fig. 2 shows the morphologies of lotus nickel using the different compounds at different Ar pressure parallel to solidification direction. Fig. 3 shows the morphologies of lotus nickel perpendicular to solidification direction. The pores of lotus-type porous nickel became elongated by unidirectional solidification. Therefore, the gas decomposed from sodium hydroxide formed homogeneous pore size and longer pore in Ar gas atmosphere. The pore size of lotus-type porous nickel using sodium hydroxide was bigger at the top than the bottom because of coalescence of the pores by evolving hydrogen gas. The pore morphology of lotus nickel became homogeneous with increasing $\mathrm{Ar}$ pressure. The pore size significantly decreases with increasing Ar pressure. Like the results of sodium hydroxide, the pores using calcium hydroxide are longer at the top. Hydrogen gas releases from the vapor forms elongated pores in $\mathrm{Ar}$ gas atmosphere by unidirectional solidification. Sodium hydroxide and calcium hydroxide decomposes to oxide particle and the vapor dissociated to hydrogen and oxygen. Therefore it is necessary to oxide particle and hydrogen for homogenous pore structure through TDM in lotus nickel. The pore size of lotus nickel decreases with increasing Ar pressure. Calcium carbonate decomposes to calcium oxide and carbon dioxide, and the decomposed carbon dioxide forms smaller pore. It results in carbon dioxide cannot dissolve in liquid metal. It can be produced smaller-sized pores using calcium carbonate decomposed into oxide and carbon dioxide. Hydrogen gas is not completely 


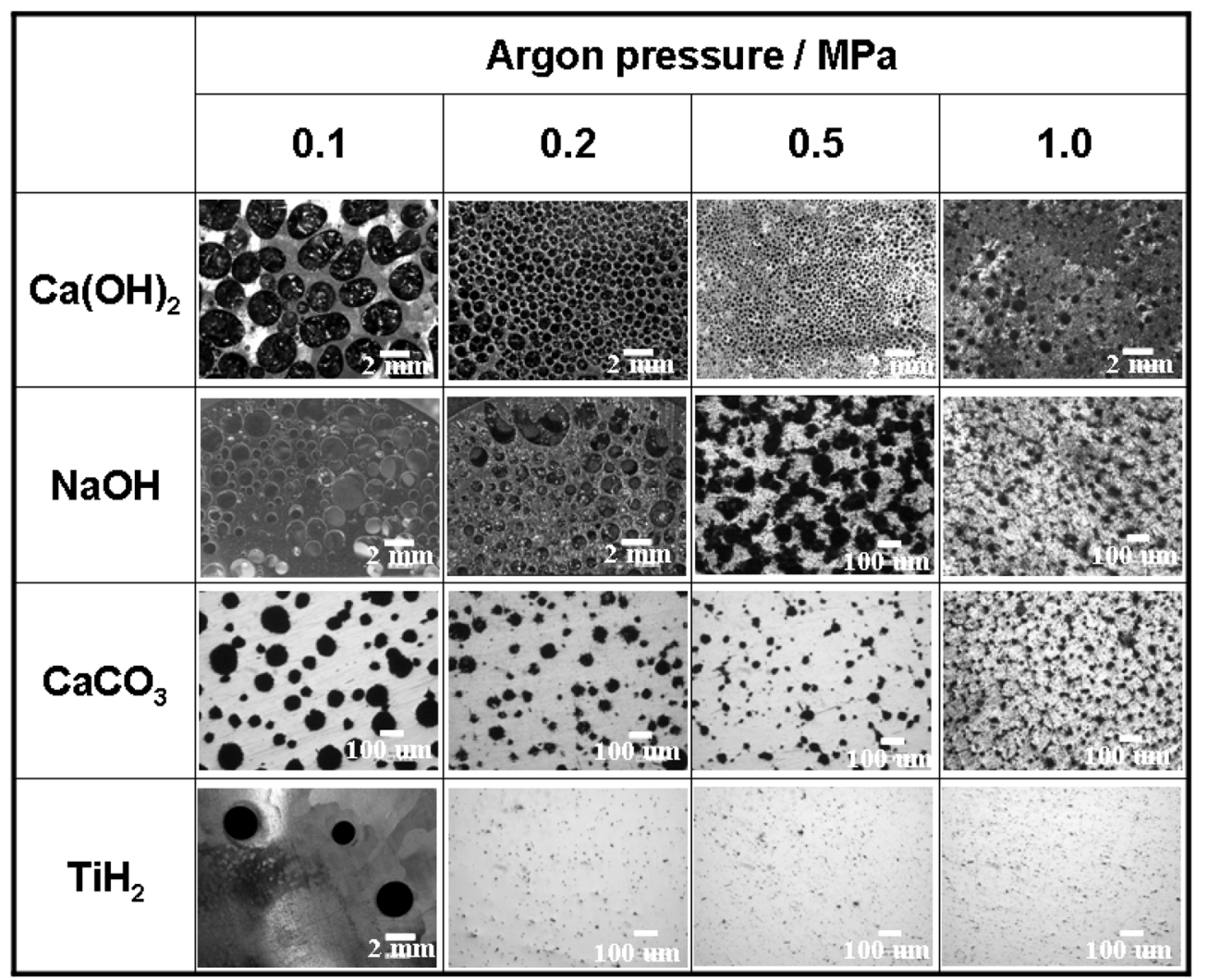

Fig. 3. Pore structure of lotus nickel fabricated through thermal decomposition perpendicular to solidification direction.

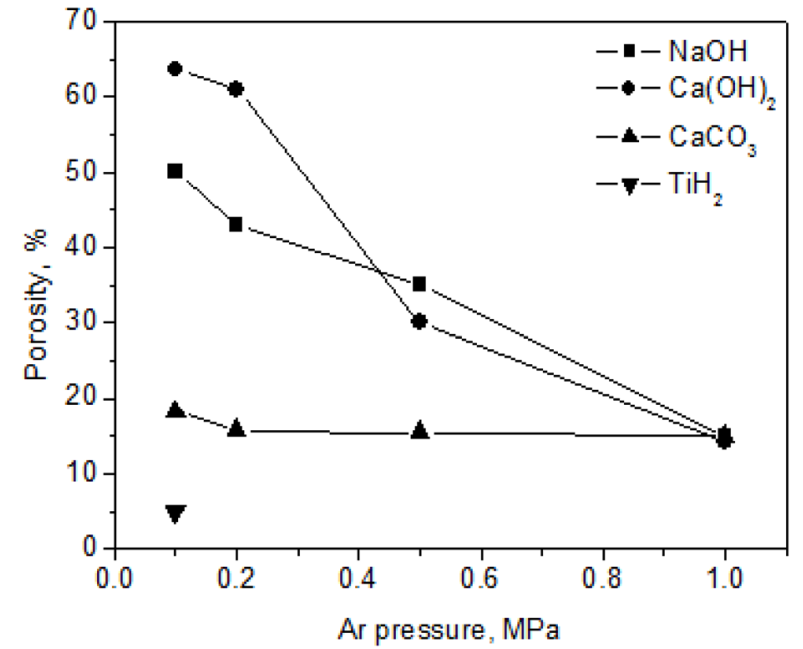

Fig. 4. The porosity of lotus-type porous nickel using different compounds with Ar atmospheric pressure.

evolved in the liquid when it is released from $\mathrm{TiH}_{2}$. Therefore, at low pressure, aligned pores form in the bottom of casting. However, at high pressure, aligned pores were not formed. Thus for titanium hydride, the pores are not formed, because the amounts of decomposed hydrogen is smaller than that of solid

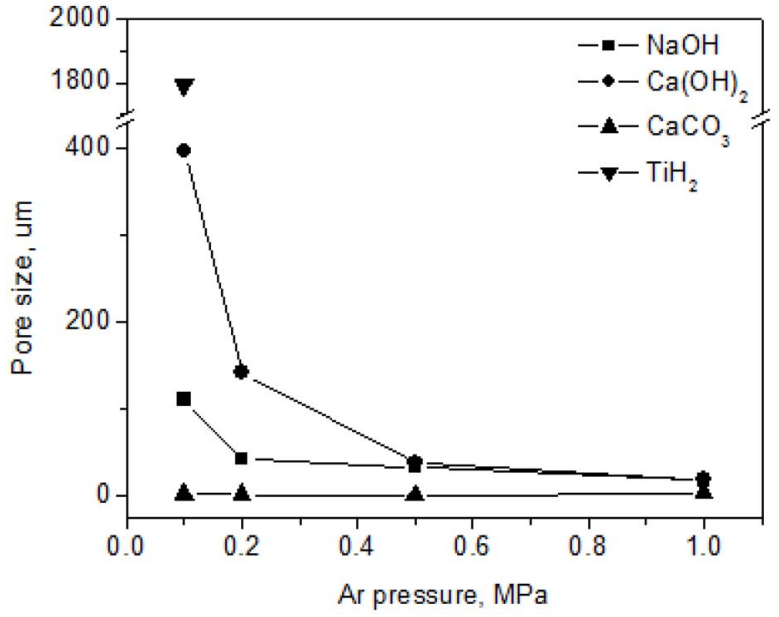

Fig. 5. The average pore size of lotus-type porous nickel using different compounds with Ar atmospheric pressure.

solubility and the pore nucleation site do not exist in the casting. It can be explained that the pores need heterogeneous nucleation for easily homogeneous pore structure formation. ${ }^{18)}$ The pore size of lotus nickel using titanium hydride is big and inhomogeneous at low pressure while the pore size is fine at high pressure. Titanium hydride decomposed to titanium and hydrogen 


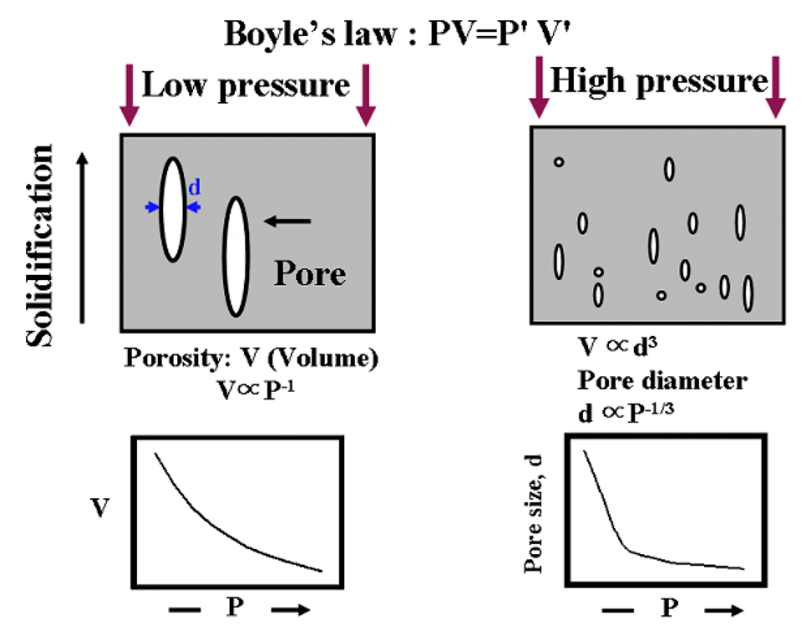

Fig. 6. Schematic drawings of pore formation mechanism in lotus nickel under Ar atmospheric pressure.

that had not oxide particle became pore nucleation site.

Fig. 4 shows the porosity of lotus nickel as a function of Ar pressure. The porosity of lotus nickel using calcium carbonate does not change with increasing $\mathrm{Ar}$ pressure, while the porosity using sodium hydroxide and calcium hydroxide significantly decreases with increasing Ar pressure. The porosity of lotus nickel does significantly change by varying atmosphere pressure because nickel has high solubility gab of hydrogen between solid and liquid state. Whereas lotus nickel using titanium hydride has low porosity at $0.1 \mathrm{MPa}$ Ar pressure, does form small and spherical pores at the higher pressure. Titanium hydride has not oxide particle when decomposes to titanium and hydrogen, the pores show the un-uniform at low pressure and very small size at high pressure.

Fig. 5 shows the variation of pore size in the lotus-type porous nickel with the pore foaming agents and the $\mathrm{Ar}$ pressure. The pore size was formed by the calcium hydroxide and the sodium hydroxide decreased with increasing the Ar pressure, although no effect was observed with the calcium carbonate. The latter was possibly due to extremely low solubility of carbon dioxide to the liquid nickel. Nevertheless, it made the pores fine in size and homogeneous in distribution through the whole samples. It means that decomposed oxide particles are play a role on pore nucleation site during solidification. It is necessary to oxide particle and the vapor which are decomposed from the compounds for forming optimum pore distribution. ${ }^{12)}$ The pore size is about $1700 \mu \mathrm{m}$ using titanium hydride that the pores are several at $0.1 \mathrm{MPa}$ due to do not contain the pore nucleation site. Over higher pressure, the pores are very small size and spherical.

Fig. 6 shows the behaviour of pore formation during solidification under Ar gas atmospheric pressure. As the Ar pressure increased, the pore size and pore structure becomes small and homogeneous. The porosity is inversely proportional to atmospheric pressure whereas the pore size is inversely proportional to cubic root of atmospheric pressure. The change of porosity and pore size can be explained by Boyle's law. The pore volume $V$, which is the porosity, is inversely proportional to the external argon pressure $P . V=n R T / P$, where $n, R$, and $T$ are the hydrogen molar number, the gas constant, and the temperature, respectively. Therefore, the pore diameter can be written as $d \propto P^{-1 / 3} \cdot{ }^{19)}$ However, the pressure dependence of the porosity and pore size cannot be explained quantitatively. It suggests that there will be another effect for suppressing the decomposition reaction with the Ar pressure.

\section{Conclusions}

Lotus nickel with long aligned pores was successfully fabricated by the mold casting technique through thermal decomposition method in Ar gas atmosphere.

Lotus nickel was fabricated by thermal decomposition method using the compounds containing gas elements. Lotus nickel with elongated pore has homogeneous pore morphology at higher $\mathrm{Ar}$ pressure. $\mathrm{H}_{2}$ gas pores are produced from $\mathrm{Ca}(\mathrm{OH})_{2}, \mathrm{NaOH}$ and $\mathrm{TiH}_{2}$, whereas $\mathrm{CO}_{2}$ is produced from $\mathrm{CaCO}_{3}$. The porosity and pore size decreases with increasing $\mathrm{Ar}$ pressure when the pores contain hydrogen. However, the porosity and pore size does not change when the pores contain carbon dioxide. The pore size and porosity of lotus nickel using compounds decreases with increasing $\mathrm{Ar}$ atmospheric pressure. It is explained by the Boyle's law that pressure of pores should be almost in balance with the ambient gas pressure.

\section{Acknowledgement}

"This work was supported by the Korea Research Foundation Grant funded by the Korean Government (MOEHRD)" (KRF-2006-352-D00090) and the Ministry of 
Education, Science Technology (MEST) and Korea Industrial Technology Foundation (KOTEF) through the Human Resource Training Project for Regional Innovation".

\section{Reference}

1. X. Badichea, S. Forest, T. Guiberta, Y. Bienveun, J.-D. Bartout, P. Ienny, M. Croset and H. Bernet, Mater. Sci. Eng. A, 289, 276 (2000).

2. J. Banhart, Prog. Mater. Sci., 46, 559 (2001).

3. S. Y. Kim, B. Y. Hur, C.K. Kwon, D. K. Ahn and S. H. Park, J. Kor. Inst. Met. \& Mater., 40, 910 (2002).

4. S. Y. Kim, H. G. Seong and B. Y. Hur, Solid State Phenomena, 124, 1801 (2007).

5. H. Nakajima, Prog. Mater. Sci., 52, 1091 (2007).

6. V. Shapovalov and L. Boyko, Adv. Mater. Eng, 6, 407 (2004).

7. Y. Liu, Y. Li, J. Wan and H. Zhang, Mater. Sci. Eng.: A, 402, 47 (2005).

8. A. E. Simone and L.J. Gibson, J. Mater. Sci., 32, 451 (1997).
9. A. E. Simone and L.J. Gibson, Acta Mater., 44, 1437 (1996).

10. S. K. Hyun, K. Murakami and H. Nakajima, Mater. Sci. Eng.: A, 299, 241 (2001).

11. T. Ide, M. Tane, S. K. Hyun and H. Nakajima, Mater. Trans., 47, 2116 (2006).

12. H. Onishi, S. K. Hyun and H. Nakajima, Mater. Trans., 47, 2120 (2006).

13. H. Nakajima and T. Ide, Metall. Mater. Trans. A, 39, 390 (2008).

14. The Wikipedia On the Web. Retrieved January 6, 2009 from http://en.wikipedia.org/wiki/ Sodium_hydroxide.

15. The Wikipedia On the Web. Retrieved January 6, 2009 from http://en.wikipedia.org/wiki/ Calcium_hydroxide.

16. D. K. Ahn, B. Y. Hur, S. Y. Kim, S. H. Park, H. J. Ahn and S. J. Park, J. Kor. Inst. Met.\& Mater., 40, 915 (2002).

17. D. H. Yang, B. Y. Hur and S. R. Yang, J. Alloy Comp., 461, 221 (2008).

18. H. Onishi, S. Ueno, S. K. Hyun and H. Nakajima, Metall. Mater. Trans. A, 40, 438 (2008).

19. S. Y. Kim, J. S. Park and H. Nakajima, Metall. Mater. Trans. A, 40, 937 (2009). 